\title{
Statistical Technique for Classification of MUAPs for Neuromuscular disease
}

\author{
Navneet Kaur \\ Deptt. of Electrical Engg., BBBSCE, \\ Fatehgarh Sahib
}

\author{
Tripatjot Singh \\ Deptt. of ECE, BBSBEC, \\ FatehgarhSsahib
}

\begin{abstract}
The Electromyograph (EMG) is useful to know the state of a patient under medical diagnosis and treatment. As the number of neuromuscular patients is increasing, it is not possible to take care of all the neuromuscular patients by carrying out manual investigations under all the conditions. Therefore it is required to design a computer aided expert system which can analyze and interpret the EMG signal. The EMG data acquisition and preprocessing, detection of MUAPs, classification of EMGs into similar groups, feature extraction of these groups and their usage in disease classification and diagnostics are the important stages in computer aided EMG analysis and interpretation. The objective of the present work is to detection of MUAPs, classification of EMGs into similar groups for computer aided analysis and interpretation of EMG signals for disease diagnosis. In the work real time recordings of myopathy, motor neuron disease and normal cases have been considered for MUAP segmentation and classification by statistical technique. EMG signal recorded by the needle electrode has been used.
\end{abstract}

\section{Keywords}

MUAP, EMG, Neuromuscular disease, segmentation

\section{INTRODUCTION}

Human body is composed of various systems and sub-systems. All human body functions are carried out by coordinated and controlled activities of the subsystems. The structural support is provided by bones while the functional support is provided by neuromuscular system. As such, the human body works under the influence of inputs from external environment and from within the body itself. Electromyography is used for the classification of various neuromuscular disorders .The word "electromyography" is made up of three parts: "electro-" + "myo-" + "-graphy." "-Myo-" is from the Greek "mys", meaning muscle and "graphy" comes from the Greek "grapho" meaning to write. So electromyography literally is the writing (recording) of muscle electricity. The bioelectric potentials associated with the muscle activity constitute the electromyogram .EMG stands for electromyography. It is the study of muscle electrical signals EMGs can be used to detect abnormal muscle electrical activity that can occur in many diseases and conditions. Electromyogram (EMG) is a test that is used to record the electrical activity of muscles. When muscles are active, they produce an electrical current. This current is usually proportional to the level of the muscle activity. The electric current may be produced due to

voluntary or involuntary muscle contraction. The EMG activity of voluntary muscle contractions is related to tension. The functional unit of the muscle contraction is a motor unit, which is comprised of a single alpha motor neuron and all the fibers it enervates. This muscle fiber contracts when the action potentials (impulse) of the motor nerve which supplies it reaches a depolarization threshold. The depolarization generates an electromagnetic field and the potential is measured as a voltage. The depolarization, which spreads along the membrane of the muscle, is a muscle action potential. The motor unit action potential is the spatial and temporal summation of the individual muscle action potentials for all the fibers of a single motor unit. Therefore, the EMG signal is the algebraic summation of the motor unit action potentials within the pick-up area of the electrode being used. The electrical signals produced by the muscles and nerves are analyzed to assess the state of neuromuscular functions in subjects with suspected neuromuscular disorders. The repetitive activation of several individual motor units results in superposed pulse train and constitutes the electromyogam signal. The potentials from individual motor units are characterized by unpredictable shapes depending upon the motor unit structure, electrode shapes and sizes, electrode placement, intervening tissues, and the state of neuromusculature. The analysis the EMG is based on its constitute i.e. motor unit action potential (MUAPs). The motor unit is the smallest functional unit of a muscle, which can be activated voluntarily. It consists of a group of muscle fibers, which are innervated from the same motor nerve. The shape of MUAP reflects the pathological and functional states of the motor unit. With increasing muscle force, the EMG signal shows an increase in the number of activated MUAPs recruited at increasing firing rate, making it difficult for the neurophysiologist to distinguish individual MUAP waveforms. In most of the clinical EMG examinations, it is the shape of the action potential that is analyzed for diagnostics. For this purpose, clinical examinations are carried out with the EMG signal recorded at low contraction level of the muscle, usually such that individual motor unit action potentials are distinguishable. This single motor unit activity is the summation of electrical activity of each muscle fiber within a motor unit. Figure1 shows the steps for the preprocessing and classification of MUAP into similar Classes.

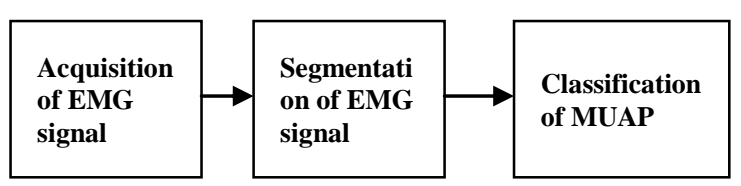

Figure-1: Block diagram of classification of EMG signal 


\section{DATA ACQUISITION}

The number of active motor units, innervation time statistics and changes in MUAP shapes during long-term measurement depend on the muscle effort, muscle fatigue, and neuromuscular dysfunction. Thus only short -term recordings of the EMG signals are usually used in clinical examination. The data base for recorded real time EMG signal was obtained from Christodulous et al. [1]. The EMG signal was acquired from the biceps and brachii muscles using a concentric needle electrode. The signal was sampled at $20 \mathrm{KHz}$ for duration of $5 \mathrm{sec}$. The MUAPs were recorded at different points in the muscle using monopolar and concentric needle electrodes in normal subjects and subjects with MYO and MND disorders.

\section{SEGMENTATION}

The information extracted from the EMG is of great importance and is used for the diagnosis and treatment of the various neuromuscular disorders and to study the neuromuscular control mechanism and muscle fatigue. Thus there is a necessity of efficient and effective techniques which can separate individual MUAPs from the complex EMG without loss of the diagnostic information. Segmentation is done to determine the number and shape of different MUAPs present, to know the approximate location of each peak and to know the amount of the MUAP waveforms throughout the EMG signal. In segmentation, the EMG is cut into the segments of possible MUAP waveforms and the low activity signal is eliminated. The segmentation technique algorithm calculates threshold depending on maximum value the complete signal. Peaks over the calculated threshold are considered as the candidate MUAPs. The threshold is taken as maximum peak/5. A window with a consent length of 120 points is taken and slide across the point, which crosses the threshold. Then this interval is examined if a peak exists in this window then the window of 120 points is taken around this peak otherwise these 120 points are saved as candidate MUAP. The threshold for the segmentation of the EMG signal is introduced to accommodate the wide range in amplitude variation in the recorded signal. The software stores only segments of data containing positive and negative peaks above a certain threshold. Determination of threshold is very critical. A lower value of the threshold may generate extra number of peaks and tend to increase the error, which mistakenly classifies the spikes belonging to same motor unit into different groups. If a higher value of threshold is chosen then it may lead to less number of groups and increase the error, which fails to distinguish the spikes of different motor units.

\section{STATISTICAL PATTERN RECOGNITION TECHNIQUE FOR CLASSIFICATION OF MUAP}

Segmented EMG signals are processed in order to identify the groups of similar MUAPs. In this work statistical pattern recognition technique has used for classification.

Statistical pattern recognition technique:-

The statistical pattern recognition technique is based on the Euclidean distance. Euclidean distance is determined to identify groups of similar waveforms using a constant threshold. The MUAP waveform that is having minimum distance or having maximum similarity from the reference waveform is considered belonging to the same class. The steps of decomposition technique are as follows: -

1. Start with first waveform, $\mathrm{x}$, as the reference waveform; being the first member of the class.

2. Calculate the Euclidean distance, $\mathrm{d}^{x}$, between the $\mathrm{x}$ and the other entire segmented waveforms y ${ }^{i}$. Calculate

$$
I_{x}=\sum_{i=1}^{n} x i^{2}
$$

Where $\mathrm{n}=400$ is the number of samples ${ }^{i}$, is the ith sample of the waveform $\mathrm{x}, 1^{x}$, is the length of the vector of waveform $\mathrm{x}$, and

$$
d x y=\sum_{i=1}^{n}(x i-y i)^{2}
$$

\section{Find those waveforms which have}

$$
d x y \leq 0.125^{*} l x
$$

If number of identified waveforms $>2$, then form a new MUAP class and calculate its average waveform and store.

4. The new reference from the remaining segments and go to step 2 and determine the Euclidean distance. If it satisfies Take the threshold criteria, then MUAP class is determined accordingly.

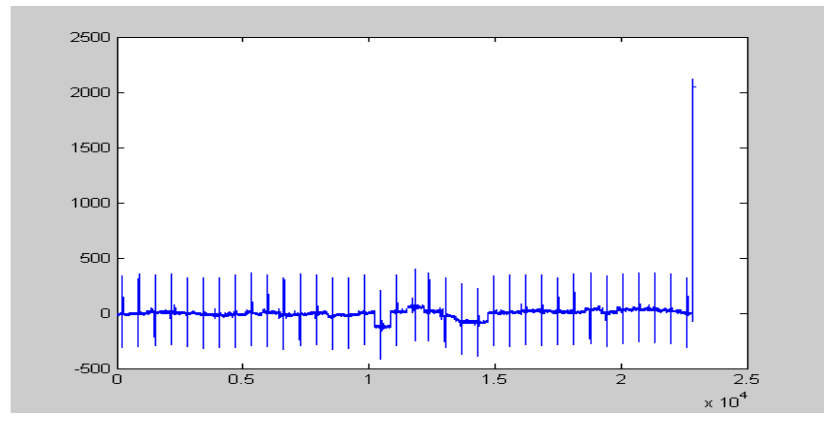

Figure 2(a) - EMG waveform of a person suffering from Myopathy (MYO)disease. 


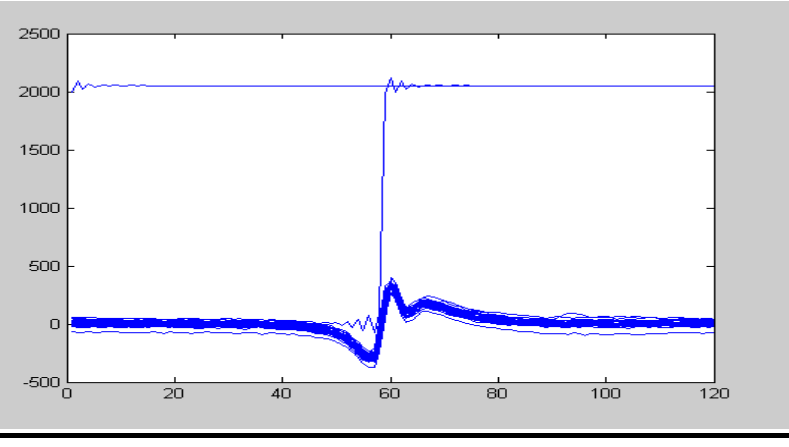

Figure 2(b) - All the segmented waves of the above MYO case (overlapped plot)

Classification of the above segmented waveform into similar groups and the average plot of each class :

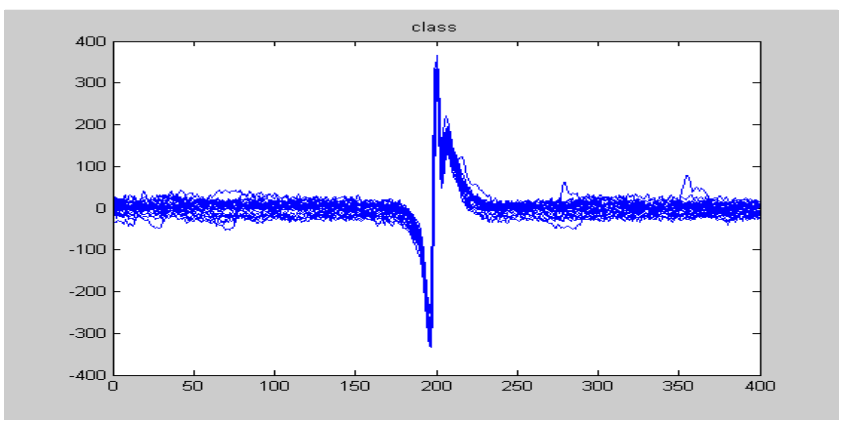

Figure 2(c).1-Class 1

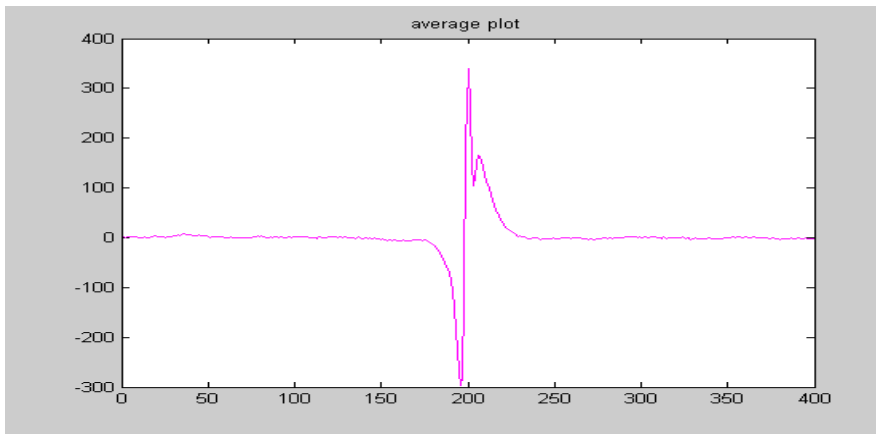

Figure 2(c).2-Average plot for class 1

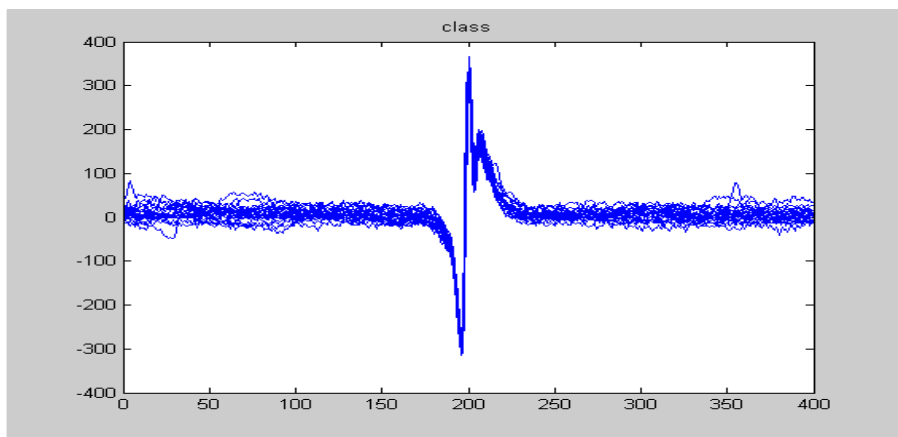

Figure 2(c).3 - Class 2

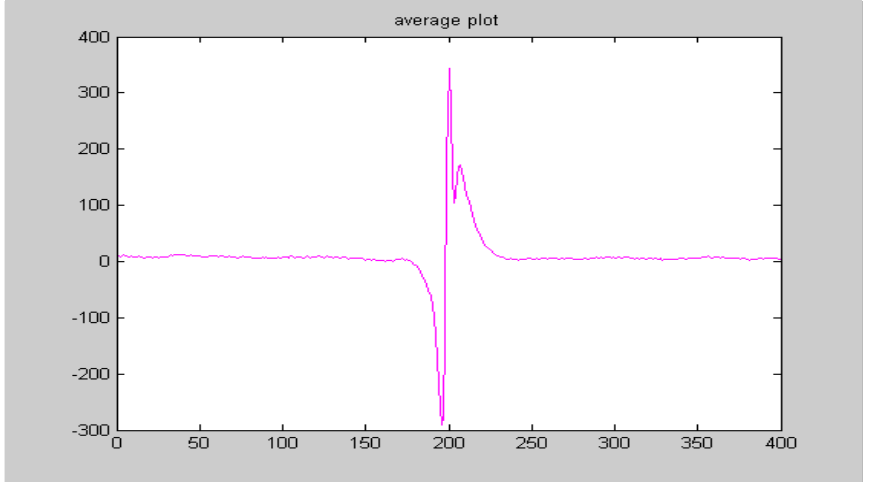

Figure 2(c).4-Average plot for class 2

\section{CONCLUSION}

The objective in this work has been to develop a expert system for analysis and interpretation of EMG signal. As the recorded EMG signal using needle electrode is composition of a large number of individual motor unit action potentials (MUAPs), necessity was felt of extracting individual clean MUAPs from the complex recorded signal using some efficient segmentation technique. After the segmentation of the recorded signal into its constitutent MUAPs, similar MUAPs have been grouped together to form the classes. For this statistical pattern recognition technique has been used which take a reference template and compare the rest with it. The results were found to be satisfactorily as compared with the available literature. 


\section{REFERENCES}

[1] Christodoulos I and Pattichis C.S., Unsupervised pattern recognition for the Classification of EMG signals", IEEE Trans. on BME Vol. 46, No.2,pp.169-178 February , 1999.

[2] Fever R.S. Le, Xenakis A.P. and Luca C.J. De. “A Procedure for decomposing the myoelectric signal into the constituent action potentials - part 2: Execution and Test for Accuracy", IEEE Trans. on BME. Vol 29, No.3, pp. 158164, March, 1982.

[3] Inbar G. F. and Noujaim A. E., "On surface EMG spectral characterization and its application to diagnostic classification", IEEE Trans. On BME, Vol, 31. No. 9, pp. 597-604, September 1984.
[4] Khandpur R.S., "Handbook of biomedical instrumentation" McGraw-hill Publishing Company Limited, New York, 1987.

[5] De Luca C.J., "Physiology and mathematics of myoelectric signals", IEEE Trans. On BME, Vol. 26, No. 6, pp 313324, June 1979.

[6] Pattichis C.S and Schizas C.N., " Genetics-based machine learning for the assessment of certain neuromuscular disorders", IEEE Trans on Neural Networks, Vol. 7, No. 2, pp. 427-436, March 1996. 